\title{
Forthcoming Behavior Research Methods, Instruments, \& Computers Articles
}

The following is a list of forthcoming Behavior Research Methods, Instruments, \& Computers articles that are currently in press. They are given in approximate order of acceptance. Each entry includes the name and address of the author with whom to communicate for further prepublication information.

"A computer-automated laboratory for studying complex perception-action skills"' by R.J. Roberts, Jr., D. Brown, S. Wiebke, \& M.M. Haith (R.J.R., Dept. of Psychology, Univ. of Denver, Denver, CO 80208)

"Word norms for blind and sighted subjects: Familiarity, concreteness, meaningfulness, imageability, imagery modality, and word associations" by N.H. Kerr \& T.H. Johnson (N.H.K., Oglethorpe Univ., 4484 Peachtree Rd., NE, Atlanta, GA 30319)

"C-QUAL: A system for computer-aided transcription of videotaped action sequences"' by S. Duncan, Jr., \& R.E. Sayre (S.D., Dept. of Psychology, Univ. of Chicago, 5848 S. University Ave., Chicago, IL 60637)

"Reinventing the algometer: Synopsis of the literature and presentation of a reliable inexpensive model" by A.J. Rainwater III \& D.W. McNeil (A.J.R., Family Medicine Residency Program, McLeod Regional Medical Ctr., 555 E. Cheves St., Florence, SC 29506)

"A VGA tachistoscope" by G.P. Finley (Dept. of Psychology, Univ. of Alberta, Edmonton, AB, Canada T6G 2E9)

"A 6809 single board computer system for the control of behavioral experiments"' by J.M. Horner (Dept. of Psychology, Colorado College, Colorado Springs, CO 80903)

"A computerized system for analyzing linear locomotion" by E. Scherer, R.E. McNicol, \& M.J. Capel (E.S., Dept. of Fisheries and Oceans, Freshwater Institute, 501 University Crescent, Winnipeg, MB, Canada R3T 2N6)
"Response key input via the IBM PC/XT/AT's parallel printer port" by E.C. Dalrymple-Alford (Dept. of Psychology, Univ. of Guelph, Guelph, ON, Canada N1G 2W1)

"NeXT in the psychology laboratory: An example of an auditory pattern tracking task" by A.J. Cohen, M. Lamoureux, \& D. Dunphy (A.J.C., Dept. of Psychology, Dalhousie Univ., Halifax, NS, Canada B3H 4J1)

"Color-vision demonstrations on an IBM PC/AT with VGA" by H. Irtel (Universität Regensburg, Institut für Psychologie, D-8400 Regensburg, Germany)

"Heuristic and statistical support for protocol analysis with SHAPA version 2.01 "' by J.M. James \& P.M. Sanderson (P.M.S., Dept. of Mech. \& Indust. Engineering, Univ. of Illinois, 1206 W. Green St., Urbana, IL 61801)

"Shuffling arrays: Appearances may be deceiving" by N.J. Castellan, Jr. (Dept. of Psychology, Indiana Univ., Bloomington, IN 47405)

"The display and animation of full-color images in real time on the Macintosh computer" by J.A. Baro \& H.C. Hughes (J.A.B., School of Optometry, Univ. of Missouri, 8001 Natural Bridge Rd., St. Louis, MO 63121-4499)

"Visual word-recognition thresholds for screen-fragmented names of the Snodgrass and Vanderwart pictures" by J.G. Snodgrass \& M. Poster (J.G.S., Dept. of Psychology, New York Univ., 6 Washington PI., Rm. 857, New York, NY 10003) 